\title{
Artificial Intelligence in the Age of Neural Networks and Brain Computing
}

\section{Edited by}

Robert Kozma

University of Memphis, Department of Mathematics, Memphis, TN, United States University of Massachusetts Amherst, Department of Computer Science, Amherst, MA, United States

Cesare Alippi

Politecnico di Milano, Milano, Italy Università della Svizzera italiana, Lugano, Switzerland

Yoonsuck Choe

Samsung Research \& Texas A\&M University, College Station, TX, United States

\section{Francesco Carlo Morabito}

University Mediterranea of Reggio Calabria, Reggio Calabria, Italy
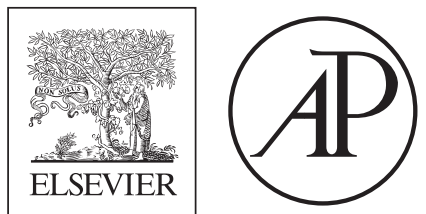

ACADEMIC PRESS

An imprint of Elsevier 


\section{Editors' Brief Biographies}

\section{Robert Kozma}

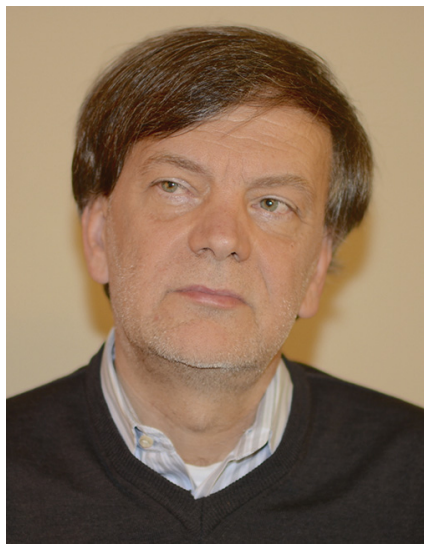

ROBERT KOZMA is a Professor of Mathematics, Director of Center for Large-Scale Integrated Optimization and Networks, University of Memphis, TN, USA; and Visiting Professor of Computer Science, University of Massachusetts Amherst. He is Fellow of IEEE and Fellow of the International Neural Network Society (INNS). He is President of INNS (2017-18) and serves on the Governing Board of IEEE Systems, Man, and Cybernetics Society (2016-18). He has served on the AdCom of the IEEE Computational Intelligence Society and on the Board of Governors of INNS. Dr. Kozma is the recipient of the INNS Gabor Award (2011) and Alumni Association Distinguished Research Achievement Award (2010). He has also served as Senior Fellow (2006-08) of US Air Force Research Laboratory. His research includes robust decision support systems, autonomous robotics and navigation, distributed sensor networks, brain networks, and braincomputer interfaces.

\section{Cesare Alippi}

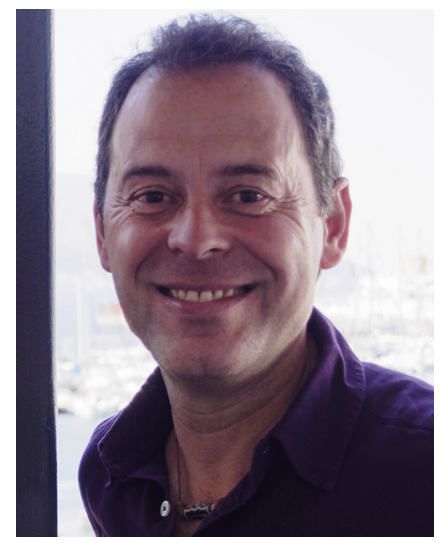

CESARE ALIPPI is a Professor with the Politecnico di Milano, Milano, Italy, and Università della Svizzera italiana, Lugano, Switzerland. $\mathrm{He}$ is an IEEE Fellow, Member of the Administrative Committee of the IEEE Computational Intelligence Society, Board of Governors member of the International Neural Network Society (INNS), and Board of Directors member of the European Neural Network Society. In 2018, he received the IEEE CIS Outstanding Computational Intelligence Magazine Award, the (2016) Gabor award from the INNS, and the IEEE Computational Intelligence Society Outstanding Transactions on Neural Networks and Learning Systems Paper Award; and in 2004, he received the IEEE Instrumentation and Measurement Society Young Engineer Award. His current research activity addresses adaptation and learning in nonstationary and time-variant environments, graphs learning, and intelligence for embedded and cyberphysical systems. 
Yoonsuck Choe

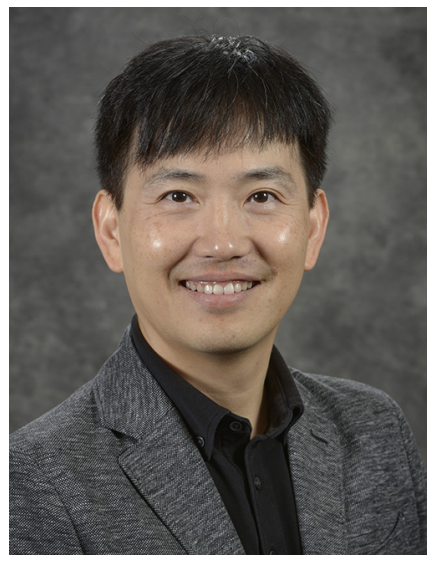

YOONSUCK CHOE is a Corporate Vice President at Samsung Research Artificial Intelligence Center (2017-present) and Professor and Director of the Brain Networks Laboratory at Texas A\&M University (2001-present). He received his $\mathrm{PhD}$ degree in computer science from the University of Texas at Austin in 2001. His research interests are in neural networks and computational neuroscience and has published over 100 papers on these topics, including a research monograph on computations in the visual cortex. He serves on the Executive Committee of the International Neural Network Society (INNS). He served as Program Chair and General Chair for IJCNN2015 and IJCNN2017, respectively, and served on the editorial board of IEEE Transactions on Neural Networks and the INNS journal Neural Networks.

Francesco Carlo Morabito

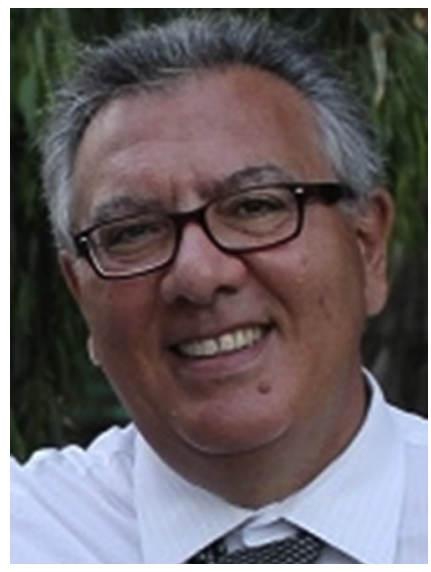

FRANCESCO CARLO MORABITO is a Professor of Electrical Engineering with the University "Mediterranea" of Reggio Calabria, Italy, and the Former Dean of the Faculty of Engineering (2001-08) and Deputy Rector of the University. He is now serving as the Vice-Rector for International Relations (2012-18). He is a Foreign Member of the Royal Academy of Doctors, Spain (2004), and Member of the Institute of Spain, Barcelona Economic Network (2017). He served as the Governor of the International Neural Network Society for 12 years and as the President of the Italian Society of Neural Networks (2008-14). He served in the organization of

IJCNN conferences (Tutorial, International Liaison, European Link, Plenary). He has coauthored over 400 papers in various fields of engineering. He is coauthor of 15 books and has three international patents. He is an Associate Editor for International Journal of Neural Systems, Neural Networks, Sensors, and Renewable Energy. 


\section{Introduction}

We live in the era of Artificial Intelligence (AI), and AI is everywhere. It is on the front page of your favorite newspaper, it is in your pocket inside your smartphone, on your kitchen table, in your car, on the street, at your office, on the trains and airplanes, everywhere. The success of AI-based commercial products, proposed by many important companies, like Google, IBM, Microsoft, Intel, Amazon, to name a few, can be interpreted as the coexistence of a successful synergism among what we call Computational Intelligence, Natural Intelligence, Brain Computing, and Neural Engineering.

The emergence of AI in many IT technologies happened almost overnight, in the past couple of years. The blessing and the curse of AI are here! And all this is just the beginning, for the better or for the worse. How did all this happen all of a sudden? Yes, it requires the powerful computing offered by advanced chips at a cheap cost. It also requires massive amount of data available through the Internet and via prolific communication resources, also called as Big Data. That is not all. Computational algorithms, called deep learning (DL), provide the framework of the programming approaches. Deep learning was coined about a decade ago, but many experts employing these technologies do not realize that DL is rooted in the technology developed by the biologically motivated neural networks field in the 1960s.

Neural networks thus powerfully reemerged with different names and meanings in different, also unexpected, contexts within the current new wave of AI and DL. Neural networks represent a well-grounded paradigm rooted in many disciplines, including computer science, physics, psychology, information science, and engineering.

This volume collects selected invited contributions from pioneers and experts of the field of neural networks. This collection aims to show that the present implications and applications of $\mathrm{AI}$ is nothing but a development of the endowed unique attributes of neural networks, namely, machine learning, distributed architectures, massively parallel processing, black-box inference, intrinsic nonlinearity, and smart autonomous search engine. We strive to cover the major basic ideas of brain-like computing behind $\mathrm{AI}$ and to contribute to give a framework to DL as well as to launch novel intriguing paradigms as possible future alternatives.

This book has been designed to commemorate the 30th anniversary of International Neural Network Society (INNS), following the 2017 International Joint Conference on Neural Networks, in Anchorage, AK, USA, May 14-18, 2017. The conference is organized jointly by the INNS and the IEEE Computational Intelligence Society (CIS), and is the premiere international meeting for researchers and other professionals in neural networks and related areas, including neural network theory, DL, computational neuroscience, robotics, and distributed intelligence.

The chapters included here are written by authors who are a blend of top experts, worldwide-recognized pioneers of the field, and researchers working on cutting-edge applications in signal processing, speech recognition, games, and adaptive control 
and decision-making. Our intent is to present the concepts involved to a target audience, who are not a narrow group of specialists working in the field but rather a broad segment of the public intrigued by recent advances in AI.

The volume presents an introduction and 15 peer-reviewed contributions briefly described in what follows.

In Chapter 1, Widrow et al. reconsider Hebbian learning, originally proposed in the field of neurobiology as one of the basis of (unsupervised) adaptive algorithms directly derived from nature. Although the LMS algorithm was previously proposed by Widrow and Hoff as a supervised learning procedure, it can be implemented in an unsupervised fashion. The two algorithms can thus be combined to form the HebbianLMS unsupervised learning algorithm, which can be the key to interpret nature's way of learning at the neuron and synapse level.

In Chapter 2, Grossberg presents a survey of the main principles, architectures, and circuits proposed by half a century of researches in the field, whose aim was to develop a unified theory of brain and mind where the psychological perspective can be read through the emergence of brain mechanisms. The chapter describes novel revolutionary paradigms, like complementary computing and laminar computing, with reference to the autonomous adaptive intelligence characteristic of the brain. The chapter reanalyzes the fundamental approach of adaptive resonance theory (ART) as a core model for engineering and technology, as well as to abstract insights into mental disorders such as autism and Alzheimer disease.

Chapter 3 is the work presented by the AI Working Group spearheaded by H. Szu and coordinated by M. Wardlaw under the aegis of the Office of Naval Research (ONR). This work provides a vista of AI from the pioneering age in the 1960s starting with narrowly defined rule-based systems through adaptive AI approaches using supervised and unsupervised neural networks. They elaborate on third generation AI based on the Zaheh-Freeman dynamic fuzzy theory, in which the Zadeh fuzzy open sets and fuzzy membership functions are not predefined; rather they evolve as the result of self-organizing recurrent chaotic neural networks, according to Freeman neurodynamics. Their approach is truly human-centered and has the premise to provide breakthroughs in AI beyond today's cutting-edge DL.

In Chapter 4, Erdi presents an insightful review on the topic of hermeneutics applied to brain science. Brain-computer-mind trichotomy is discussed, where downward causality is discussed as a unique feature of brain-mind as opposed to computation. Hermeneutics is introduced next, applied to the brain, and it is argued that the brain is in principle a hermeneutic device. One application of this idea is the explanation of schizophrenia, which is argued to be due to a broken hermeneutic cycle. Finally, the chapter concludes with thoughts on how to achieve algorithms for neural/mental hermeneutics. This is a deep theoretical essay that touches upon fundamental issues in brain and neural sciences.

In Chapter 5, Ormandy addresses crucial issues related to the limitations of mainstream AI and neural network technologies, especially in the context of the usefulness of the AI in developing new technologies to improve our quality of life. He describes the work started in collaboration with the late Walter Freeman in order to capture 
the dynamics of embodied human cognition and incorporate it to novel wearable personal assistants. The author reviews the literature from file theories through embodied cognition across species. The main thesis of this work is the critical importance of ephaptic interactions between neural populations, which produce neural fields measurable by noninvasive means, thus providing an opportunity for the development of wearable personal assistants in everyday life, including augmented memory, stress relief, fitness training, relaxation, and other applications.

In Chapter 6, Kasabov presents an approach based on evolutionary connectionist systems (ECOS) that are able to evolve their architecture and functionality in adaptive data-driven modality. Evolving spiking neural networks (eSNN) are illustrated and proposed as a third generation of artificial neural networks (ANN). eSNN can be used for future brain-like AI, and the NeuCube architecture is presented as a machine that can implement DL procedures. The chapter also proposes a combination of $\mathrm{AI}$ and $\mathrm{ANN}$ approaches as a unique method derived from neuroscience.

In Chapter 7, Brown et al. focused on the pitfalls and opportunities of developing techniques of evaluation of AI systems. This is a cool topic, considering the relevant progresses that DL methodologies have introduced in the computational intelligence community. However, they raise the problem of measuring and comparing performance. The receiver operating characteristic (ROC) paradigm and the bootstrap method are considered as well-grounded approaches for performance metric in order to avoid overestimation that frequently limits the practical implementations of AI.

In Chapter 8, Werbos provides an impressive summary and a critical review of the reasons underlying today's wave of AI successes, including DL and the Internet-ofThings (IoT). As a major exponent both in research and research funding in the past decades, he provides an exciting insider's view of these developments, as well as points toward possible avenues in future research, neuroscience in particular. He also points out the key role researchers play in applying the novel technological development for the benefit of humanity.

In Chapter 9, Levine reviews the history of neural networks as an artificial model of brain and mind. Neural networks are a paradigm that in principle link biology and technology: it thus comes as no surprise that the flagship journal of the International Neural Network Society is indeed Neural Networks. His chapter reconsiders the original ideas that motivate the nascence of this interdisciplinary society at the light of present developments.

In Chapter 10, Kozma touches a fundamental problem humans have focused on for centuries, that of creating machines that act like them. The chapter investigates various aspects of biological and $\mathrm{AI}$ issues and introduces a balanced approach based on the concepts of complementarity and multistability as manifested in human brain operation and cognitive processing. As intelligence in human brains is the result of a delicate balance between fragmentation of local components and global dominance of coherent overall states, the chapter elaborates on how intelligence is manifested through the emergence of a sequence of coherent metastable amplitude patterns. This behavior leads to the cinematic theory of human cognition that both provides 
insights into key principles of intelligence in biological brains and helps in building more powerful artificially intelligent devices.

In Chapter 11, Morabito et al. introduce a comprehensive investigation of DL applications in brain engineering and biomedical signal processing, with a particular focus on the processing of multivariate time-series coming from electrophysiology. Electroencephalography (EEG) and high density EEG and magnetoencephalography technologies are reviewed, as they constitute the measurement systems yielding multivariate electrophysiological time-series. The use of DL methods for multivariate EEG time-series processing are then detailed to permit the reader to easily enter this fascinating application framework. Future direction of research in DL, encompassing interpretability, architectures and learning procedures, and robustness aspects are then discussed, so as to provide the reader with some relevant open research topics.

In Chapter 12, Alippi et al. present a timely and thorough review on the use of computational intelligence and machine learning methods in cyber-physical systems (CPS) and IoT. The review goes over four major research topics in this domain: (1) system architecture; (2) energy harvesting, conservation, and management; (3) fault detection and mitigation; and (4) cyberattack detection and countermeasures. Importantly, the authors challenge assumptions that are taken for granted but do not apply anymore to the increasingly more complex CPS and IoT systems. These assumptions include high energy availability, stationarity, correct data availability, and security guarantees. This chapter provides an excellent review of the status of CPS and IoT and how to overcome the issues in these emerging fields.

In Chapter 13, Tagliaferri et al. present an innovative approach of multiview learning as a branch of machine learning for the analysis of multimodal data in biomedical applications, among which, in particular, the authors focus on bioinformatics (i.e., gene expression, microRNA expression, protein-protein interactions, genome-wide association). The approach proposed allows capturing information regarding different aspects of the underlying principles governing complex biological systems. The authors also propose an example of how both clustering and classification can be combined in a multiview setting for the automated diagnosis of neurodegenerative disorders. They also show using some application examples how recent DL techniques can be applied to multimodal data to learn complex representations.

In Chapter 14, Choe provides educated discussions and analyses about some key concepts central to brain science and AI, namely those associated with the dichotomies: meaning vs. information, prediction vs. memory, and question vs. answer. The author shows how a slightly different view on these concepts can help us move forward, beyond current limits of our understanding in these fields. In detail, the chapter elaborates over the intriguing definition of information as seen from different perspectives and its relationship with the concept of meaning. Then, it investigates the role of plasticity, that of memory, and how they relate to prediction. Finally, the focus moves on to the question vs. answer issue in AI algorithms and how it impacts on their ability to solve problems. 
In Chapter 15, Miikkulainen et al. present a novel automated method for designing deep neural network architecture. The main idea is based on neuroevolution to evolve the neural network topology and parameters. In the proposed work, neuroevolution is extended to evolve topology, components (modules), and hyperparameters. The method is applied to both feedforward architectures like CNN and also to recurrent neural networks (with LSTM units). The proposed method is tested on standard image tasks (object recognition) and natural language tasks (image captioning), demonstrating comparable results as state-of-the-art methods. This chapter provides a great overview of evolutionary methods developed at Sentient technologies for the design and optimization of deep neural networks. 


\section{List of Contributors}

\section{Cesare Alippi}

Politecnico di Milano, Milano, Italy; Università della Svizzera Italiana, Lugano, Switzerland

\section{David G. Brown}

US Food and Drug Administration, Silver Spring, MD, United States

\section{Maurizio Campolo}

NeuroLab, DICEAM, University Mediterranea of Reggio Calabria, Reggio

Calabria, Italy

\section{Yoonsuck Choe}

Samsung Research, Seoul, Korea; Department of Computer Science and Engineering, Texas A\&M University

\section{Nigel Duffy}

Sentient Technologies, Inc., San Francisco, CA, United States

\section{Péter Érdi}

Center for Complex Systems Studies, Kalamazoo College, Kalamazoo, MI, United States; Institute for Particle and Nuclear Physics, Wigner Research Centre for Physics, Hungarian Academy of Sciences, Budapest, Hungary

\section{Daniel Fink}

Sentient Technologies, Inc., San Francisco, CA, United States

\section{Olivier Francon}

Sentient Technologies, Inc., San Francisco, CA, United States

\section{Paola Galdi}

NeuRoNe Lab, DISA-MIS, University of Salerno, Fisciano, Italy

\section{Stephen Grossberg}

Center for Adaptive Systems Graduate Program in Cognitive and Neural Systems, Departments of Mathematics \& Statistics, Psychological \& Brain Sciences, and Biomedical Engineering, Boston University, Boston, MA, United States

\section{Babak Hodjat}

Sentient Technologies, Inc., San Francisco, CA, United States

\section{Cosimo leracitano}

NeuroLab, DICEAM, University Mediterranea of Reggio Calabria, Reggio

Calabria, Italy

\section{Nikola Kasabov}

Knowledge Engineering and Discovery Research Institute - KEDRI, Auckland University of Technology, Auckland, New Zealand 


\section{Youngsik Kim}

Department of Electrical Engineering, Stanford University, Stanford, CA, United States

\section{Robert Kozma}

University of Memphis, Department of Mathematics, Memphis, TN, United States; University of Massachusetts Amherst, Department of Computer Science, Amherst, MA, United States

\section{Daniel S. Levine}

University of Texas at Arlington, Arlington, TX, United States

\section{Jason Liang}

Sentient Technologies, Inc., San Francisco, CA, United States; The University of Texas at Austin, Austin, TX, United States

\section{Nadia Mammone}

NeuroLab, DICEAM, University Mediterranea of Reggio Calabria, Reggio Calabria, Italy

\section{Elliot Meyerson}

Sentient Technologies, Inc., San Francisco, CA, United States; The University of Texas at Austin, Austin, TX, United States

\section{Risto Miikkulainen}

Sentient Technologies, Inc., San Francisco, CA, United States; The University of Texas at Austin, Austin, TX, United States

\section{Francesco Carlo Morabito}

NeuroLab, DICEAM, University Mediterranea of Reggio Calabria, Reggio Calabria, Italy

\section{Arshak Navruzyan}

Sentient Technologies, Inc., San Francisco, CA, United States

\section{Roman Ormandy}

Embody Corporation, Los Gatos, CA, United States

\section{Seiichi Ozawa}

Kobe University, Kobe, Japan

\section{Dookun Park}

Department of Electrical Engineering, Stanford University, Stanford, CA, United States

\section{Jose Krause Perin}

Department of Electrical Engineering, Stanford University, Stanford, CA, United States

\section{Bala Raju}

Sentient Technologies, Inc., San Francisco, CA, United States 


\section{Aditya Rawal}

Sentient Technologies, Inc., San Francisco, CA, United States; The University of Texas at Austin, Austin, TX, United States

\section{Frank W. Samuelson}

US Food and Drug Administration, Silver Spring, MD, United States

\section{Angela Serra}

NeuRoNe Lab, DISA-MIS, University of Salerno, Fisciano, Italy

\section{Hormoz Shahrzad}

Sentient Technologies, Inc., San Francisco, CA, United States

\section{Harold Szu}

Catholic University of America, Washington, DC, United States

\section{Roberto Tagliaferri}

NeuRoNe Lab, DISA-MIS, University of Salerno, Fisciano, Italy

\section{The Al Working Group}

Catholic University of America, Washington, DC, United States

\section{Paul J. Werbos}

US National Science Foundation, retired, and IntControl LLC, Arlington, VA, United States

\section{Bernard Widrow}

Department of Electrical Engineering, Stanford University, Stanford, CA, United States 